Pacific Journal of Mathematics

EXTREMAL SPECTRAL FUNCTIONS OF A SYMMETRIC 


\title{
EXTREMAL SPECTRAL FUNCTIONS OF A SYMMETRIC OPERATOR
}

\author{
RichaRd C. GilberT
}

1. Introduction. Let $H_{1}$ be a symmetric operator in a Hilbert space $\mathfrak{S}_{1}$. If $H$ is a self-adjoint operator in a Hilbert space $\mathfrak{S}$ such that $\mathfrak{S}_{1} \subset \mathfrak{S}$ and $H_{1} \subset H$, then $H$ is called a self-adjoint extension of $H_{1}$. If $\mathfrak{S}_{2} \ominus \mathfrak{S}_{1}$ is finite-dimensional, then $H$ is called a finite-dimensional self-adjoint extension of $H_{1} . \quad H$ is called a minimal self-adjoint extension if neither $\mathfrak{S}_{2} \ominus \mathfrak{S}_{1}$ nor any of its subspaces different from $\{0\}$ reduces $H$.

Suppose $H$ is a self-adjoint extension of $H_{1}$. If $E(\lambda)$ is the spectral function of $H$ and if $P_{1}$ is the operator in $\mathfrak{L}_{2}$ of orthogonal projection on $\mathfrak{S}_{1}$, then the operator function $E_{1}(\lambda)=P_{1} E(\lambda)$ restricted to $\mathfrak{F}_{1}$ is called a spectral function of $H_{1}$. We shall say that the spectral function $E_{1}(\lambda)$ is defined by the self-adjoint extension $H$.

The family of spectral functions of $H_{1}$ is a convex set, i.e., if $E_{1}^{\prime}(\lambda)$ and $E_{1}^{\prime \prime}(\lambda)$ are spectral functions of $H_{1}$ and if $a$ and $b$ are nonnegative real numbers such that $a+b=1$, then $a E_{1}^{\prime}(\lambda)+b E_{1}^{\prime \prime}(\lambda)$ is also a spectral function of $H_{1}$. A spectral function $E_{1}(\lambda)$ of $H_{1}$ is said to be extremal if it is impossible to find two different spectral functions $E_{1}^{\prime}(\lambda), E_{1}^{\prime \prime}(\lambda)$ and positive real numbers $a$ and $b, a+b=1$, such that $E_{1}(\lambda)=a E_{1}^{\prime}(\lambda)+b E_{1}^{\prime \prime}(\lambda)$.

For further information we refer the reader to Achieser and Glasmann [1].

M. A. Naimark [6] has shown that the finite-dimensional extensions of a symmetric operator define extremal spectral functions of the operator. Finite-dimensional extensions exist, however, only for symmetric operators with equal deficiency indices. In $\S 4$ of this paper it is shown that self-adjoint extensions defined by the addition of maximal symmetric operators determine extremal spectral functions for a symmetric operator with unequal deficiency indices. The proof uses the proposition of M. A. Naimark [6] that if $E_{1}(\lambda)$ is defined by the minimal self-adjoint extension $H$, then $E_{1}(\lambda)$ is extremal if and only if every bounded self-adjoint operator $A$ which commutes with $H$ and satisfies the condition $(A f, g)=(f, g)$ for all $f, g \in \mathfrak{K}_{1}$ is reduced by $\mathfrak{S}_{1}$. Section 2 is devoted to a description of the self-adjoint extensions of a symmetric operator, and section 3 identifies some extremal spectral functions of a symmetric operator with infinite equal deficiency indices other than the ones defined by finite-dimensional extensions.

Received May 15, 1963. This work was supported by the Mathematics Research Center, U.S. Army, Madison, Wisconsin, under Contract No.: DA-11-022-ORD-2059. 
The proof is based on the proposition of M. A. Naimark mentioned above.

2. Self-adjoint extensions of a symmetric operator. The linear operator $H$ in the Hilbert space $\mathfrak{S}$ is said to be Hermitian if $(H f, g)=$ $(f, H g)$ for all $f, g \in \mathfrak{D}(H) . \quad H$ is symmetric if it is Hermitian and $\overline{\mathfrak{D}(H)}=\mathfrak{S}$. If $H$ is a closed Hermitian operator and $\lambda$ is a nonreal number, we define the subspaces $\mathfrak{M}(\lambda)$ and $\mathscr{L}(\lambda)$ by the equations $\mathscr{L}(\lambda)=$ $\mathfrak{R}(H-\bar{\lambda} E)$ and $\mathfrak{M}(\lambda)=\mathfrak{S} \ominus \mathfrak{L}(\lambda)$. ( $E$ stands for the identity operator.) $\mathfrak{M}(\lambda)$ is called a deficiency subspace of $H$ and has the same dimensions for all $\lambda$ in the same half-plane (upper or lower.) If $m=\operatorname{dim} \mathfrak{M}(\bar{\lambda})$, $n=\operatorname{dim} \mathfrak{M}(\lambda)$, then $(m, n)$ are called the deficiency indices of $H$ (with respect to $\lambda$ ). (We add "with respect to $\lambda$ " because the ordered pair $(m, n)$ depends on the half-plane $\lambda$ is in.) The operator $U(\lambda)=$ $(H-\bar{\lambda} E)(H-\lambda E)^{-1}$ is an isometry mapping $\mathscr{L}(\bar{\lambda})$ onto $\mathcal{L}(\lambda)$. It is called the Cayley transform of $H$. We have that $H=$ $(\lambda U(\lambda)-\bar{\lambda} E)(U(\lambda)-E)^{-1}$. Since $\lambda$ is a fixed non-real number in the following, we shall write $U$ in place of $U(\lambda)$. For fixed $\lambda$ the correspondence between a Hermitian operator and its Cayley transform is a one-to-one inclusion-preserving correspondence between the set of closed Hermitian operators $H$ and the set of closed isometric operators $U$ for which $(U-E)^{-1}$ exists. We note, finally, that a subspace $\mathfrak{S}_{1}$ reduces $H$ if and only if $\mathfrak{S}_{1}$ reduces $U$. In this circumstance, if $\mathfrak{S}_{2}=\mathfrak{S}_{2} \ominus \mathfrak{S}_{1}$, and if $H_{i}$ and $U_{i}$ are $H$ and $U$ respectively restricted to $\mathfrak{S}_{i}$, then $U_{i}$ is the Cayley transform of $H_{i}$ and $H=H_{1} \oplus H_{2}, U=U_{1} \oplus U_{2}$.

M. A. Naimark [5] has proved the following theorem which describes all self-adjoint extensions of a symmetric operator.

THEOREM 1. Let $\lambda$ be any fixed nonreal number. Let $H_{1}$ be a closed symmetric operator with deficiency indices $\left(m_{1}, n_{1}\right)$ (with respect to $\lambda$ ). Then every self-adjoint extension $H$ of $H_{1}$ is obtained as follows:

(1) Let $\mathrm{H}_{2}$ be a closed Hermitian operator in $\mathfrak{K}_{2}$ with deficiency indices $\left(m_{2}, n_{2}\right)$ (with respect to $\lambda$ ) satisfying $m_{1}+m_{2}=n_{1}+n_{2}$, $m_{2} \leqq n_{1}$.

(2) Let $H_{0}=H_{1} \oplus H_{2}$ in $\mathfrak{S}=\mathfrak{S}_{1} \oplus \mathfrak{S}_{2}$. $\quad\left(H_{0}\right.$ is therefore a closed Hermitian operator with equal deficiency indices $\left(m_{1}+m_{2}, n_{1}+n_{2}\right)$, and if $U_{i}$ is the Cayley transform of $H_{i}, i=0,1,2$, then $U_{0}=U_{1} \oplus U_{2}$. Further, $\left.\mathfrak{M}_{0}(\bar{\lambda})=\mathfrak{M}_{1}(\bar{\lambda}) \oplus \mathfrak{M}_{2}(\bar{\lambda}), \mathfrak{M}_{0}(\lambda)=\mathfrak{M}_{1}(\lambda) \oplus \mathfrak{M}_{2}(\lambda)\right)$.

(3) Let $V$ be an arbitrary isometric operator mapping $\mathfrak{M}_{0}(\bar{\lambda})$ onto $\mathfrak{M}_{0}(\lambda)$ satisfying the condition $\varphi \in \mathfrak{M}_{2}(\bar{\lambda}), \quad V \varphi \in \mathfrak{M}_{2}(\lambda)$ implies $\varphi=0$.

(4) Let $\mathfrak{D}(H)$ be defined as all $g=f+V \varphi-\varphi$, where $f \in \mathfrak{D}\left(H_{0}\right)$, $\varphi \in \mathfrak{M}_{0}(\bar{\lambda})$. 
(5) If $g \in \mathfrak{D}(H)$, let $H g=H_{0} f+\lambda V \varphi-\bar{\lambda} \varphi$.

Then, $H$ is self-adjoint extension in $\mathfrak{S}$ of $H_{1}$, and every selfadjoint extension of $H_{1}$ is obtained in this way. We have that $\mathfrak{D}\left(H_{2}\right)=\mathfrak{D}(H) \cap \mathfrak{S}_{2}$.

We say that $H_{2}$ and $V$ of Theorem 1 define the self-adjoint extension $H$ of $H_{1}$.

We can put the operator $V$ into correspondence with a matrix $\left(V_{i k}\right)$ of operators such that $V_{11}: \mathfrak{M}_{1}(\bar{\lambda}) \rightarrow \mathfrak{M}_{1}(\lambda), \quad V_{12}: \mathfrak{M}_{2}(\bar{\lambda}) \rightarrow \mathfrak{M}_{1}(\lambda)$, $V_{21}: \mathfrak{M}_{1}(\bar{\lambda}) \rightarrow \mathfrak{M}_{2}(\lambda), \quad V_{22}: \mathfrak{M}_{2}(\bar{\lambda}) \rightarrow \mathfrak{M}_{2}(\lambda)$. Then condition on $V$ in (3) of theorem 1 then becomes $V_{12} \varphi=0$ implies $\varphi=0$.

We now give a theorem which gives a more detailed analysis of the structure of $V$.

THeOREM 2. Suppose that $\mathfrak{M}_{1}(\lambda), \mathfrak{M}_{1}(\bar{\lambda}), \mathfrak{M}_{2}(\lambda), \mathfrak{M}_{2}(\bar{\lambda})$ are Hilbert spaces and that $V$ is an isometry which maps $\mathfrak{M}_{1}(\bar{\lambda}) \oplus \mathfrak{M}_{2}(\bar{\lambda})$ onto $\mathfrak{M}_{1}(\lambda) \oplus \mathfrak{M}_{2}(\lambda)$. ( $\lambda$ here has nothing to do with the theorem and is retained only as a notational convenience.) If $V=\left(V_{i k}\right)$ in matrix form, suppose that $V_{12} \varphi=0$ implies that $\varphi=0$. Then the following conclusions are true:

(1) If $\mathfrak{M}_{1}^{-}(\lambda)$ is defined by the equation $\mathfrak{M}_{1}^{-}(\lambda)=\left[V_{12} \mathfrak{M}_{2}(\bar{\lambda})\right]^{c}(c$ indicates closure of a set) and if $\mathfrak{N}_{1}(\lambda)$ is defined by $\mathfrak{N}_{1}(\lambda)=$ $\mathfrak{M}_{1}(\lambda) \ominus \mathfrak{M}_{1}^{-}(\lambda)$, then $\mathfrak{R}_{1}(\lambda)$ is the null space of $V_{12}^{*}$. Thus, $V_{12}^{*}$ is one-to-one on $\mathfrak{M}_{1}^{-}(\lambda)$. Further, $\mathfrak{M q}_{2}(\bar{\lambda})=\left[V_{12}^{*} \mathfrak{M}_{1}^{-}(\lambda)\right]^{c}$.

(2) $V^{*}=V^{-1}$ maps $\mathfrak{R}_{1}(\lambda)$ onto a subspaces of $\mathfrak{M}_{1}(\bar{\lambda})$, which we

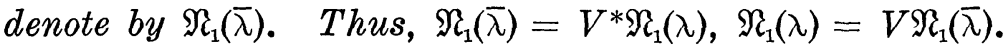

(3) If $\mathfrak{M}_{1}^{-}(\bar{\lambda})$ is defined by the equation $\mathfrak{M}_{1}^{-}(\bar{\lambda})=\mathfrak{M}_{1}(\bar{\lambda}) \ominus \mathfrak{M}_{1}(\bar{\lambda})$, then $V$ maps $\mathfrak{M}_{1}^{-}(\bar{\lambda}) \oplus \mathfrak{M}_{2}(\bar{\lambda})$ isometrically onto $\mathfrak{M}_{1}^{-}(\lambda) \oplus \mathfrak{M}_{2}(\lambda)$.

Thus, $V_{11} \mathfrak{M}_{1}^{-}(\bar{\lambda}) \subset \mathfrak{M}_{1}^{--}(\lambda)$.

(4) $V_{21}$ is one-to-one on $\mathfrak{M}_{1}^{-}(\bar{\lambda})$, and $\mathfrak{N}_{1}(\bar{\lambda})$ is the null space of $V_{21} \cdot \mathfrak{M}_{2}(\lambda)=\left[V_{21} \mathfrak{M}_{1}^{-}(\bar{\lambda})\right]^{c}$.

(5) $\quad V_{21}^{*}$ is one-to-one on $\mathfrak{M}_{2}(\lambda)$ and $\mathfrak{M}_{1}^{-}(\bar{\lambda})=\left[V_{21}^{*} \mathfrak{M R}_{2}(\lambda)\right]^{c}$.

(6) If $m_{1}=\operatorname{dim} \mathfrak{M}_{1}(\bar{\lambda}), \quad n_{1}=\operatorname{dim} \mathfrak{M}_{1}(\lambda), \quad m_{2}=\operatorname{dim} \mathfrak{M}_{2}(\bar{\lambda}), \quad n_{2}=$ $\operatorname{dim} \mathfrak{M}_{2}(\lambda)$, then $m_{1}+m_{2}=n_{1}+n_{2}, m_{2}=\operatorname{dim} \mathfrak{M}_{2}(\bar{\lambda})=\operatorname{dim} \mathfrak{M}_{1}^{-}(\lambda) \leqq n_{1}$, $n_{2}=\operatorname{dim} \mathfrak{M k}_{2}(\lambda)=\operatorname{dim} \mathfrak{M}_{1}^{-}(\bar{\lambda}) \leqq m_{1}$.

(7) If $m_{2}=n_{2}, m_{1}=n_{1}$.

Proof. (1) Since $\mathfrak{N}_{1}(\lambda)$ is the orthogonal complement of the closure of the range of $V_{12}, \mathfrak{R}_{1}(\lambda)$ is the null space of $V_{12}^{*}$, and $V_{12}^{*}$ is one-to-one on $\mathfrak{M}_{1}^{-}(\lambda)$.

Suppose $g \in \mathfrak{M l}_{2}(\bar{\lambda})$ and $g$ is perpendicular to $V_{12}^{*} \mathfrak{M}_{1}^{-}(\lambda)$. Then $0=$ $\left(g, V_{12}^{*} f\right)=\left(V_{12} g, f\right)$ for all $f \in \mathfrak{M}_{1}^{-}(\lambda)$. Therefore, $V_{12} g=0$, and, since $V_{12}$ is one-to-one, $g=0$. Thus, $M_{2}(\bar{\lambda})=\left[V_{12}^{*} \mathfrak{M}_{1}^{-}(\lambda)\right]^{c}$. 
(2) Since

$$
V^{*}=\left(\begin{array}{ll}
V_{11}^{*} & V_{21}^{*} \\
V_{12}^{*} & V_{22}^{*}
\end{array}\right),
$$

$V^{*} \mathfrak{N}_{1}(\lambda)=V_{11}^{*} \mathfrak{N}_{1}(\lambda) \subset \mathfrak{M}_{1}(\bar{\lambda}) . \quad$ Thus, $V^{*}=V^{-1}$ maps $\mathfrak{R}_{1}(\lambda)$ onto a subspace of $\mathfrak{M}_{1}(\bar{\lambda})$.

(3) Clear, since $\mathfrak{N}_{1}(\lambda)=V \mathfrak{N}_{1}(\bar{\lambda})$.

(4) We first show that $V_{21}$ is one-to-one on $\mathfrak{M}_{1}^{-}(\bar{\lambda})$. Suppose $f \in \mathfrak{M}_{1}^{-}(\bar{\lambda}), \quad V_{21} f=0$. Then, $\quad V f=V_{11} f+V_{21} f=V_{11} f \in \mathfrak{M}_{1}^{-}(\lambda)$. Let $g=V_{11} f=V f$, so that $f=V^{*} g=V_{11}^{*} g+V_{12}^{*} g$. Since $f \in \mathfrak{M}_{1}^{-}(\bar{\lambda})$, $V_{11}^{*} g \in \mathfrak{M}_{1}^{-}(\bar{\lambda}), \quad V_{12}^{*} g \in \mathfrak{M}_{2}(\bar{\lambda})$, we have that $V_{12}^{*} g=0$. By (1) and the fact that $g \in \mathfrak{M}_{1}^{-}(\lambda), g=0$. Thus, $f=V^{*} g=0$, and our contention is proved.

Since $\mathfrak{N}_{1}(\lambda)=V \mathfrak{N}_{1}(\bar{\lambda}), \quad V_{21} f=0$ for all $f \in \mathfrak{N}_{1}(\bar{\lambda})$. On the other hand, we have just shown that $V_{21}$ is one-to-one on $\mathfrak{M}_{1}^{-}(\bar{\lambda})$. It follows that $\mathfrak{R}_{1}(\bar{\lambda})$ is the null space of $V_{21}$.

Because $\left(V_{21}^{*}\right)^{*}=V_{21}$ and the null space of $\left(V_{21}^{*}\right)^{*}$ is the orthogonal complement of the closure of the range of $V_{21}^{*}$, we see that $\mathfrak{M}_{1}^{-}(\bar{\lambda})=$ $\left[V_{21}^{*} \mathfrak{M}_{2}(\lambda)\right]^{c}$.

We claim finally that $\mathfrak{M}_{2}(\lambda)=\left[V_{21} M_{1}^{-}(\bar{\lambda})\right]^{c}$. Suppose $g \in \mathfrak{M}_{2}(\lambda)$ and that $g$ is perpendicular to $V_{21} \mathfrak{M}_{1}^{-}(\bar{\lambda})$. Therefore, $0=\left(V_{21} f, g\right)=\left(f, V_{21}^{*} g\right)$ for all $f \in \mathfrak{M}_{1}^{-}(\bar{\lambda})$. Since $V_{21}^{*} g \in \mathfrak{M}_{1}^{-}(\bar{\lambda})$, it follows that $V_{21}^{*} g=0$. Thus, $V^{*} g=V_{22}^{*} g \in \mathfrak{M}_{2}(\bar{\lambda})$. Let $f=V^{*} g$. Then, $g=V f=V_{12} f+V_{22} f$, where $g \in \mathfrak{M}_{2}(\lambda), V_{12} f \in \mathfrak{M}_{1}^{-}(\lambda), V_{22} f \in \mathfrak{M}_{2}(\lambda)$. Hence, $V_{12} f=0$ and $f=0$. Whence, $g=V f=0$. This proves our claim and completes the proof of (4).

(5) We have already shown in (4) that $\mathfrak{M}_{1}^{-}(\bar{\lambda})=\left[V_{21}^{*} \mathfrak{M}_{2}(\lambda)\right]^{c}$. Since we also showed in (4) that $\mathfrak{M}_{2}(\lambda)=\left[V_{21} \mathfrak{M}_{1}^{-}(\bar{\lambda})\right]^{c}$, it follows that the null space of $V_{21}^{*}$ is empty and therefore $V_{21}^{*}$ is one-to-one on $\mathfrak{M R}_{2}(\lambda)$.

(6) $m_{1}+m_{2}=n_{1}+n_{2}$ follows from the fact that $V$ maps $\mathfrak{M}_{1}(\bar{\lambda}) \oplus \mathfrak{M}_{2}(\bar{\lambda})$ isometrically onto $\mathfrak{M}_{1}(\lambda) \oplus \mathfrak{M}_{2}(\lambda)$.

We claim now that $\operatorname{dim} \mathfrak{M}_{2}(\bar{\lambda})=\operatorname{dim} \mathfrak{M}_{1}^{-}(\lambda)$. Let $\left\{\varphi_{\alpha}\right\}$ be a complete orthonormal system in $\mathfrak{M}_{2}(\bar{\lambda})$. Then $\left\{V_{12} \varphi_{a}\right\}$ is a fundamental set in $\mathfrak{M}_{1}^{-}(\lambda)$. (See Nagy [4] for definitions.) Therefore $\operatorname{dim} \mathfrak{M}_{2}(\bar{\lambda})=P\left\{\varphi_{\alpha}\right\}=$ $P\left\{V_{12} \varphi_{a}\right\} \geqq \operatorname{dim} \mathfrak{M}_{1}^{-}(\lambda)$, where $P$ stands for cardinality. Using $V_{12}^{*}$ and an analogous argument, we obtain that $\operatorname{dim} \mathfrak{M}_{1}^{-}(\lambda) \geqq \operatorname{dim} \mathfrak{M}_{2}(\bar{\lambda})$. Thus, $\operatorname{dim} \mathfrak{M}_{2}(\bar{\lambda})=\operatorname{dim} \mathfrak{M}_{1}^{-}(\lambda)$, and $m_{2}=\operatorname{dim} \mathfrak{M}_{2}(\bar{\lambda})=\operatorname{dim} \mathfrak{M}_{1}^{-}(\lambda) \leqq n_{1} . \quad$ Similarly, $n_{2}=\operatorname{dim} \mathfrak{M R}_{2}(\lambda)=\operatorname{dim} \mathfrak{M}_{1}^{-}(\bar{\lambda}) \leqq m_{1}$.

(7) The proof is clear from the inequalities in (6).

Theorem 2 is therefore completely proved.

Theorem 3. (M. A. Naimark [5]). For each self-adjoint extension $H$ in $\mathfrak{S}$ of a symmetric operator $H_{1}$ in $\mathfrak{S}_{1}$ there exists a minimal self-adjoint extension $H_{0}$ in $\mathfrak{S}_{0}$ such that

(1) $\mathfrak{S}_{1} \subset \mathfrak{S}_{0} \subset \mathfrak{2}$; 
(2) $H_{1} \subset H_{0} \subset H$;

(3) $H_{0}$ and $H$ define the same spectral function of $H_{1}$.

THEOREM 4. Suppose that $H_{1}$ is a closed symmetric operator and that $H_{2}$ and $V$ define a self-adjoint extension $H$ of $H_{1}$. Let $H_{0}$ be a self-adjoint extension of $H_{1}$ having the properties that $\mathfrak{S}_{1} \subset \mathfrak{S}_{0} \subset \mathfrak{K}_{2}$ and $H_{1} \subset H_{0} \subset H$. Then the following statements are true:

(1) If we write $\mathfrak{S}_{0}=\mathfrak{S}_{1} \oplus \mathfrak{S}_{3}, \mathfrak{S}_{2}=\mathfrak{S}_{0} \oplus \mathfrak{S}_{4}=\mathfrak{S}_{1} \oplus \mathfrak{S}_{3} \oplus \mathfrak{S}_{4}, \mathfrak{K}_{2}=$ $\mathfrak{S}_{3} \oplus \mathfrak{S}_{4}$, then $H$ is reduced by $\mathfrak{S}_{4}$ and $H=H_{0} \oplus H_{4}$, where $H_{4}$ is a self-adjoint operator in $\mathfrak{S}_{4}$.

(2) $\mathfrak{S}_{4} \subset \mathfrak{L}_{2}(\bar{\lambda}) \cap \mathfrak{R}_{2}(\lambda), \mathfrak{M}_{2}(\bar{\lambda}) \subset \mathfrak{S}_{3}, \mathfrak{M}_{2}(\lambda) \subset \mathfrak{S}_{3}$.

(3) $H_{2}$ is reduced by $\mathfrak{S}_{4}$ and $H_{2}=H_{3} \oplus H_{4}$, where $H_{3}$ is a closed Hermitian operator in $\mathfrak{S}_{3}$ with the same deficiency subspaces $M_{2}(\bar{\lambda})$, $\mathfrak{M}_{2}(\lambda)$ as $H_{2}$.

(4) $H_{0}$ is defined by $H_{3}$ and $V$.

(5) $H$ and $H_{0}$ define the same spectral function of $H_{1}$.

Proof. (1) Since $H_{1} \subset H_{0} \subset H$, we have that $U_{1} \subset U_{0} \subset U$. Because $U_{0}$ maps $\mathfrak{K}_{0}$ isometrically onto $\mathfrak{S}_{0}$ and $U$ maps $\mathfrak{K}$ isometrically onto $\mathfrak{S}_{2}$, we have that $U$ maps $\mathfrak{S}_{4}$ isometrically onto $\mathfrak{S}_{4}$. Thus, $\mathfrak{S}_{4}$ reduces $U$, and hence $U=U_{0} \oplus U_{4}, H=H_{0} \oplus H_{4}$, where $H_{4}$ is a selfadjoint operator in $\mathfrak{S}_{4}$ with Cayley transform $U_{4}$. This proves (1).

(2) We claim first that $\mathfrak{S}_{4} \subset \mathfrak{R}_{2}(\bar{\lambda})$. Let $f \in \mathfrak{K}_{4}$. Since $H_{4} \subset \mathfrak{K}_{2}=$ $\mathfrak{M}_{2}(\bar{\lambda}) \oplus \mathfrak{R}_{2}(\bar{\lambda}), f=f^{\prime}+f^{\prime \prime}$, where $f^{\prime} \in \mathfrak{M L}_{2}(\bar{\lambda}), f^{\prime \prime} \in \mathbb{R}_{2}(\bar{\lambda})$. Hence, $U f=$ $U f^{\prime}+U f^{\prime \prime}=V f^{\prime}+U_{2} f^{\prime \prime}=V_{12} f^{\prime}+V_{22} f^{\prime}+U_{2} f^{\prime \prime}$, where $U f \in \mathfrak{K}_{4} \subset \mathfrak{K}_{2}$, $V_{12} f^{\prime} \in \mathfrak{M}_{1}(\lambda) \subset \mathfrak{S}_{1}, V_{22} f^{\prime} \in \mathfrak{M}_{2}(\lambda) \subset \mathfrak{S}_{2}, U_{2} f^{\prime \prime} \in \mathfrak{R}_{2}(\lambda) \subset \mathfrak{K}_{2}$. Thus, $V_{12} f^{\prime}=$ 0 , and therefore $f^{\prime}=0$. It follows that $f=f^{\prime \prime} \in \mathfrak{R}_{2}(\bar{\lambda})$ and that $\mathfrak{S}_{4} \subset \mathbb{R}_{2}(\bar{\lambda})$.

Since $\mathfrak{S}_{4} \subset \mathfrak{S}_{2}(\bar{\lambda})$, and since $U$ maps $\mathfrak{K}_{4}$ isometrically onto $\mathfrak{K}_{4}$ and $\mathfrak{R}_{2}(\bar{\lambda})$ isometrically onto $\mathfrak{R}_{2}(\lambda)$, we conclude that $\mathfrak{g}_{4} \subset \mathfrak{R}_{2}(\lambda)$. Hence, $\mathfrak{S}_{4} \subset \mathfrak{R}_{2}(\bar{\lambda}) \cap \mathfrak{R}_{2}(\lambda)$. It follows immediately that $\mathfrak{M}_{2}(\bar{\lambda}) \subset \mathfrak{S}_{3}, \mathfrak{M}_{2}(\lambda) \subset \mathfrak{S}_{3}$. (2) is therefore completely proved.

(3) Because $U_{2}=U$ on $\mathscr{L}_{2}(\bar{\lambda})$, we see that $U_{2}$ maps $\mathfrak{S}_{4}$ isometrically onto $\mathfrak{K}_{4}$. We know, however, that $U_{2}$ maps $\mathfrak{L}_{2}(\bar{\lambda})$ isometrically onto $\Re_{2}(\lambda)$. It follows that $\mathfrak{S}_{4}$ reduces $U_{2}$. Thus, $U_{2}=U_{3} \oplus U_{4}$, where $U_{3}$ maps $\mathfrak{R}_{2}(\bar{\lambda}) \ominus \mathfrak{K}_{4}$ isometrically onto $\mathfrak{R}_{2}(\lambda) \ominus \mathfrak{K}_{4}$, and $H_{2}=H_{3} \oplus H_{4}$, where $H_{3}$ is a closed Hermitian operator in $\mathfrak{S}_{3}$ with Cayley transform $U_{3}$. Noting that $\mathfrak{S}_{3}=\mathfrak{M}_{2}(\lambda) \oplus\left[\mathfrak{R}_{2}(\bar{\lambda}) \ominus \mathfrak{S}_{4}\right]=\mathfrak{M}_{2}(\lambda) \oplus\left[\mathcal{R}_{2}(\lambda) \ominus \mathfrak{S}_{4}\right]$, we see that $H_{3}$ has deficiency subspaces $\mathfrak{M}_{2}(\bar{\lambda}), \mathfrak{M}_{2}(\lambda)$. This proves (3).

(4) By Theorem $1, H_{3}$ and $V$ define a self-adjoint extension $H_{0}^{\prime}$ of $H_{1}$ in $\mathfrak{S}_{0}=\mathfrak{S}_{1} \oplus \mathfrak{S}_{3}$. If $U_{0}^{\prime}$ is the Cayley transform of $H_{0}^{\prime}$, then $U_{0}^{\prime}=U_{1}=U$ on $\mathfrak{R}_{1}(\bar{\lambda}), \quad U_{0}^{\prime}=V=U$ on $\mathfrak{M}_{1}(\bar{\lambda}) \oplus \mathfrak{M}_{2}(\bar{\lambda}), \quad U_{0}^{\prime}=U_{3}=U$ on $\mathfrak{R}_{2}(\bar{\lambda}) \ominus \mathfrak{S}_{4}$. It follows that $U_{0}^{\prime}=U$ on $\mathfrak{S}_{1} \oplus \mathfrak{K}_{3}=\mathfrak{K}_{0}$. But since $U_{0} \subset U, U_{0}=U$ on $\mathfrak{S}_{0}$, hence, $U_{0}=U_{0}^{\prime}$, and therefore $H_{0}=H_{0}^{\prime}$. This 
proves (4).

(5) As we have shown, $H=H_{0} \oplus H_{4}$. Thus, $E(\lambda)=E_{0}(\lambda) \oplus E_{4}(\lambda)$, and therefore $E(\lambda) f=E_{0}(\lambda) f$ for all $f \in \mathfrak{S}_{1}$. If $P$ is the operator of orthogonal projection of $\mathfrak{S}_{2}$ onto $\mathfrak{S}_{1}$ and if $P_{0}$ is the operator of orthogonal projection of $\mathfrak{S}_{0}$ onto $\mathfrak{S}_{1}, P E(\lambda) f=P E_{0}(\lambda) f=P_{0} E_{0}(\lambda) f$ for all $f \in \mathfrak{S}_{1}$, so that $H$ and $H_{0}$ define the same spectral function of $H_{1}$. This proves (5), and the proof of theorem 4 is completed.

3. Extremal spectral functions of a symmetric operator with equal deficiency indices.

THEOREM 5. Let $H$ be a self-adjoint extension of the closed symmetric operator $H_{1}$. Suppose that $H$ is defined by $H_{2}$ and $V$. Then the following statements are equivalent:

(1) $\mathfrak{D}\left(H_{2}\right)=\{0\}$.

(2) $\mathfrak{M}_{2}(\bar{\lambda})=\mathfrak{M}_{2}(\lambda)=\mathfrak{S}_{2}$.

(3) $\mathfrak{D}(H) \cap \mathfrak{K}_{2}=\{0\}$.

Proof. That (1) implies (2) is clear from the definition of $\mathfrak{M l}_{2}(\bar{\lambda})$ and $\mathfrak{M}_{2}(\lambda)$. Suppose, on the other hand, that $\mathfrak{M}_{2}(\bar{\lambda})=\mathfrak{M}_{2}(\lambda)=\mathfrak{K}_{2}$. Then, $\Re\left(H_{2}-\lambda E\right)=\mathfrak{R}\left(H_{2}-\bar{\lambda} E\right)=\{0\}$. If $f \in \mathfrak{D}\left(H_{2}\right), H_{2} f-\lambda f=0$ and $H_{2} f-\bar{\lambda} f=0$. Subtracting the first equation from the second, $(\lambda-\bar{\lambda}) f=0$, and therefore $f=0$. Thus, $\mathfrak{D}\left(H_{2}\right)=\{0\}$, and we have proved that (2) implies (1).

By Theorem 1, $\mathfrak{D}\left(H_{2}\right)=\mathfrak{D}(H) \cap \mathfrak{S}_{2}$, so that (1) and (3) are clearly equivalent.

THEOREM 6. Let $H_{1}$ be a closed symmetric operator. Suppose that $H$ is a self-adjoint extension of $H_{1}$ defined by $H_{2}$ and $V$. If $\mathfrak{D}\left(H_{2}\right)=\{0\}$, the following statements are true:

(1) $m_{1}=n_{1}$, i.e., the deficiency indices of $H_{1}$ are equal.

(2) $H$ is minimal.

(3) The spectral function $E_{1}(\lambda)$ of $H_{1}$ defined by $H$ is extremal.

Proof. (1) By Theorem 5, $\mathfrak{D}\left(H_{2}\right)=\{0\}$ implies that $m_{2}=n_{2}$. By theorem 2, (7), $m_{1}=n_{1}$.

(2) By Theorem 5, $\mathfrak{D}\left(H_{2}\right)=\{0\}$ implies that $\mathfrak{M}_{2}(\bar{\lambda})=\mathfrak{M}_{2}(\lambda)=\mathfrak{S}_{2}$. Hence, $\mathfrak{\Omega}_{2}(\bar{\lambda})=\mathfrak{\Omega}_{2}(\lambda)=\{0\}$. It follows from Theorem 3 and Theorem 4 , (2), that $H$ is minimal.

(3) Let $A$ be any bounded operator in $\mathfrak{S}$ having a matrix representation,

$$
A \sim\left(\begin{array}{ll}
E & B \\
B^{*} & C
\end{array}\right)
$$


where $E$ is the identity in $\mathfrak{S}_{1}, B$ maps $\mathfrak{S}_{2}$ into $\mathfrak{S}_{1}, C$ maps $\mathfrak{S}_{2}$ into $\mathfrak{K}_{2}$, and $C$ is self-adjoint. Suppose that $A$ commutes with $H$. We shall show that this implies that $B \equiv 0$. By the proposition of M. A. Naimark [6] mentioned in the introduction, then, it follows that the spectral function $E_{1}(\lambda)$ defined by $H$ is extremal.

Since $A$ commutes with $H$, it commutes with the Cayley transform $U$ of $H$. If we represent $U$ as a matrix, $U \sim\left(U_{j k}\right)$, where $U_{j k}$ maps $\mathfrak{S}_{k}$ into $\mathfrak{S}_{j}$, then the fact that $A$ commutes with $U$ implies that $B U_{21}=U_{12} B^{*}$. Taking adjoints, we also have that $U_{21}^{*} B^{*}=B U_{12}^{*}$. We observe, further, that $U=V$ on $\mathfrak{M}_{1}(\bar{\lambda}) \oplus \mathfrak{M}_{2}(\bar{\lambda})$ and that $U^{*}=$ $U^{-1}=V^{-1}=V^{*}$ on $\mathfrak{M}_{1}(\lambda) \oplus \mathfrak{M}_{2}(\lambda)$.

Using the equation $B U_{12}^{*}=U_{21}^{*} B^{*}$, the fact that $\mathfrak{M}_{2}(\lambda)=\mathfrak{L}_{2}$, and Theorem 2, we obtain that $B V_{22}^{*} \mathfrak{M}_{1}^{-}(\lambda)=B U_{12}^{*} \mathbb{M}_{1}^{-}(\lambda)=U_{21}^{*} B^{*} \mathfrak{M}_{1}^{-}(\lambda) \subset U_{21}^{*} \mathcal{K}_{2}=$ $U_{21}^{*} \mathfrak{M}_{2}(\lambda)=V_{21}^{*} \mathfrak{M}_{2}(\lambda) \subset \mathfrak{M}_{1}(\bar{\lambda})$. Since by Theorem $2 V_{12}^{*} \mathfrak{M}_{1}^{-}(\lambda)$ is dense in $M_{2}(\bar{\lambda})=\mathfrak{K}_{2}$ and since $B$ is bounded, it follows that $B \mathfrak{S}_{2} \subset \mathfrak{M R}_{1}(\bar{\lambda})$.

Similarly, using the equation $B U_{21}=U_{12} B^{*}$, we obtain that $B V_{21} \mathfrak{M}_{1}^{-}(\bar{\lambda})=B U_{21} \mathfrak{M}_{1}^{-}(\bar{\lambda})=U_{12} B^{*} \mathfrak{M}_{1}^{-}(\bar{\lambda}) \subset U_{12} \mathfrak{S}_{2}=U_{12} \mathfrak{M}_{2}(\bar{\lambda})=V_{12} \mathfrak{M R}_{2}(\bar{\lambda}) \subset$ $\mathfrak{M}_{1}(\lambda)$, and therefore $B \mathfrak{S}_{2} \subset \mathfrak{M}_{1}(\lambda)$.

Thus, $B \mathfrak{C}_{2} \subset \mathfrak{M}_{1}(\bar{\lambda}) \cap \mathfrak{M}_{1}(\lambda)$. But $\mathfrak{M}_{1}(\bar{\lambda}) \cap \mathfrak{M}_{1}(\lambda)=\{0\}$, because $\mathfrak{M}_{1}(\bar{\lambda})$ and $\mathfrak{M}_{1}(\lambda)$ are the deficiency subspaces of a symmetric operator. Hence, $B \equiv 0$. This complete the proof of Theorem 6 .

By use of a somewhat less general form of Theorem 6, M. A. Naimark [6] has shown that every finite-dimensional extension $H$ of a closed symmetric operator $H_{1}$ defines an extremal spectral function of $H_{1}$.

Theorem 7. If $H$ is a finite-dimensional extension of a closed symmetric operator $H_{1}$, then $H_{1}$ must have equal deficiency indices.

Proof. Suppose that $H$ is defined by $H_{2}$ and $V$. Then $H_{2}$ is a Hermitian operator in the finite-dimensional space $\mathfrak{K}_{2}$. Since $U_{2}$ maps $\Re_{2}(\bar{\lambda})$ isometrically onto $\Re_{2}(\lambda)$, it follows that $\operatorname{dim} \Omega_{2}(\bar{\lambda})=\operatorname{dim} \Re_{2}(\lambda)$. Hence $\operatorname{dim} \mathfrak{M l}_{2}(\bar{\lambda})=\operatorname{dim} \mathfrak{M}_{2}(\lambda)$, i.e., $m_{2}=n_{2}$. By Theorem 2, (7), $m_{1}=$ $n_{1}$. This proves Theorem 7 .

4. Extremal spectral functions of a symmetric operator with unequal deficiency indices. We first introduce the notion of a partial isometry and some of the properties thereof. (See Murray and von Neumann [3].) A bounded linear operator $W$ in a Hilbert space $\mathfrak{F}$ is called a partial isometry if it maps a subspace $\&$ isometrically onto another subspace $\mathfrak{F}$, while it maps $\mathfrak{S} \ominus \mathbb{F}$ onto $\{0\}$. \& is called the initial set of $W$, and $\mathfrak{F}$ is called the final set of $W$. If $W$ is a partial isometry, then the following statements hold:

(1) If $P(\xi)$ is the operator of orthogonal projection on $\&$ and if $P(\mathfrak{F})$ is the operator of orthogonal projection on $\widetilde{f}$, then $P(\mathfrak{F})=W^{*} W^{*}$; 
$P(\mathfrak{F})=W W^{*}$

(2) $U^{*}$ is a partial isometry with initial set $\mathfrak{F}$ and final set $\mathfrak{F}$.

(3) As a mapping of $\mathfrak{F}$ onto $\mathfrak{F}, U^{*}$ is the inverse of $U$ as a mapping of $\mathbb{F}$ onto $\mathfrak{F}$.

THEOREM 8. Suppose that $W$ is a partial isometry with initial set $\mathfrak{M}$ and final set $\mathfrak{S}$. Let $\mathfrak{N}=\mathfrak{K} \ominus \mathfrak{M}$. Then, $\mathfrak{M}=\mathfrak{M}^{\prime} \oplus \mathfrak{M} \mathfrak{N}^{\prime \prime}$, where

(1) W maps $\mathfrak{M}^{\prime \prime}$ isometrically onto $\mathfrak{M}^{\prime \prime}$;

(2) if $f \in \mathfrak{R} \oplus \mathfrak{M}^{\prime}, \lim _{p \rightarrow \infty} W^{p} f=0$.

Proof. Let $\mathfrak{M}_{i}=\left(W^{*}\right)^{i \mathfrak{N},} i=0,1,2, \cdots$. Then each $\mathfrak{M}_{i}$ is a subspace (i.e., a closed linear manifold), and the following statements are true:

(a) $\mathfrak{M}_{i} \subset \mathfrak{M}$ for $i=1,2, \cdots$. This is clear because $W^{*}$ is a partial isometry with initial set $\mathfrak{S}$ and final set $\mathfrak{M}$.

(b) If $f \in \mathfrak{M}_{n}$, where $n \geqq 0$, then $W^{p} f \in \mathfrak{M}_{n-p}$ for $1 \leqq p \leqq n$, and $W^{p} f=0$ for $p>n$. Proof: If $f \in \mathfrak{M}_{n}$, then $f=\left(W^{*}\right)^{n} g$ for some $g \in \mathfrak{N}$. Since $W W^{*}=E, W^{p} f=\left(W^{*}\right)^{n-p} g \in \mathfrak{M}_{n-p}, 1 \leqq p \leqq n$. If $p>n, W^{p} f=$ $W^{p-n} g=0$.

(c) If $f \in \mathfrak{M}_{i}, i=0,1,2, \cdots$, and if $n$ is a positive integer, then $\left(W^{*}\right)^{n} f \in \mathfrak{M}_{i+n}$. Proof: If $f \in \mathfrak{M}_{i}, f=\left(W^{*}\right)^{i} g$, where $g \in \mathfrak{N}$. Therefore, $\left(W^{*}\right)^{n} f=\left(W^{*}\right)^{i+n} g \in \mathfrak{M}_{i+n}$.

(d) $\mathfrak{M}_{i}$ is perpendicular to $\mathfrak{M}_{j}$ if $i \neq j$. Proof: Suppose $i<j$, and let $f \in \mathfrak{M}_{i}, g \in \mathfrak{M}_{j}$. Then there exists $f_{1} \in \mathfrak{N}$ and $g_{1} \in \mathfrak{N}$ such that $f=\left(W^{*}\right)^{i} f_{1}, \quad g=\left(W^{*}\right)^{j} g_{1}$. Hence, $(f, g)=\left(\left(W^{*}\right)^{i} f_{1},\left(W^{*}\right)^{j} g_{1}\right)=$ $\left(f_{1},\left(W^{*}\right)^{j-i} g_{1}\right)=0$, since $f_{1} \in \mathfrak{R},\left(W^{*}\right)^{j-i} g_{1} \in \mathfrak{M}_{j-i} \subset \mathfrak{M}$.

Now let $\mathfrak{M I}^{\prime}=\sum_{i=1}^{\infty} \mathfrak{M}_{i}$. Then $\mathfrak{M}^{\prime}$ is a subspace of $\mathfrak{M}$. Let $\mathfrak{M}^{\prime \prime}=\mathfrak{M} \ominus \mathfrak{M}^{\prime}$. We shall show that $\mathfrak{M}^{\prime}$ and $\mathfrak{M}^{\prime \prime}$ satisfy (1) and (2).

Since $\mathfrak{M}=\mathfrak{M}^{\prime} \oplus \mathfrak{M}^{\prime \prime}$ and $\mathfrak{S}=\mathfrak{N} \oplus \mathfrak{M}^{\prime} \oplus \mathfrak{M}^{\prime \prime}$, and since $W$ maps $\mathfrak{M}$ isometrically onto $\mathfrak{E}$, in order to prove (1) it is sufficient to show that $W$ maps $\mathfrak{M E}^{\prime}$ onto $\mathfrak{R} \oplus \mathfrak{M}^{\prime}$. Suppose $f \in \mathfrak{M}^{\prime}$. Then, $f=\sum_{i=1}^{\infty} f_{i}$, where $f_{i} \in \mathfrak{M}_{i}$, and $W f=\sum_{i=1}^{\infty} W f_{i}$. Because by (b) $W f_{i} \in \mathfrak{M}_{i-1}$, we see that $W f \in \mathfrak{R} \oplus \mathfrak{M}^{\prime}$. Thus, $W$ maps $\mathfrak{M}^{\prime}$ into $\mathfrak{N} \oplus \mathfrak{M} \mathfrak{M}^{\prime}$. To show that the map is onto, let $g \in \mathfrak{N} \oplus \mathfrak{M}^{\prime}$. Then, $g=\sum_{i=0}^{\infty} f_{i}$, where $f_{i} \in \mathfrak{M}_{i}$. If $f=W^{*} f=\sum_{i=0}^{\infty} W^{*} f_{i} \in \mathfrak{M}^{\prime}$, by (c). Further, $W f=W W^{*} g=g$. Hence, $W$ maps $\mathfrak{M}^{\prime}$ onto $\mathfrak{R} \oplus \mathfrak{M}^{\prime}$.

We now prove (2). Let $f \in \mathfrak{R} \oplus \mathfrak{M R}^{\prime}$. Then, $f=\sum_{i=0}^{\infty} f_{i}$, where $f_{i} \in \mathfrak{M}_{i}$. By (b), $W^{p} f=\sum_{i=0}^{\infty} W^{p} f_{i}=\sum_{i=p}^{\infty} W^{p} f_{i}$. Hence, $\left\|W^{p} f\right\|^{2}=$ $\sum_{i=p}^{\infty}\left\|W^{p} f_{i}\right\|^{2}=\sum_{i=p}^{\infty}\left\|f_{i}\right\|^{2}$. Thus, $\lim _{p \rightarrow \infty}\left\|W^{p} f\right\|^{2}=0$. This proves (2) and completes the proof of the theorem.

THeOREM 9. Let $\lambda$ be a fixed nonreal number. Suppose that $H_{1}$ is a closed symmetric operator in $\mathfrak{S}_{1}$ with deficiency indices $(m, n)$ 
(with respect to $\lambda$ ), and suppose that $m \neq n$. Let $H$ be a self-adjoint extension of $H_{1}$ defined by $H_{2}$ and $V$, where $H_{2}$ is a closed Hermitian operator with deficiency indices $(0, s), n+s=m$, if $m>n$ and $(s, 0), m+s=n$, if $m<n$. Then the spectral function defined by $H$ is extremal.

Proof. Assume that $m>n$. The case $m<n$ then follows by interchanging the roles of $\bar{\lambda}$ and $\lambda$ in Theorem 1 and defining $H$ by $H_{2}$ and $V^{*}$.

By Theorem 3 there exists a minimal self-adjoint extension $H_{0}$ of $H_{1}$ such that $\mathfrak{S}_{1} \subset \mathfrak{S}_{0} \subset \mathfrak{S}_{2}, H_{1} \subset H_{0} \subset H$, and $H_{0}$ and $H$ define the same spectral function of $H_{1}$. By Theorem $4, H_{0}$ is defined by $V$ and a Hermitian operator $H_{3}$ with the same deficiency subspaces as $H_{2}$. Since we can always consider $H_{0}$ instead of $H$, it follows that without loss of generality we can consider $H$ to be a minimal self-adjoint extension.

Since $\mathfrak{M}_{2}(\bar{\lambda})=\{0\} \quad$ and $\mathfrak{L}_{2}(\bar{\lambda})=\mathfrak{S}_{2}$, we have that if $f \in \mathfrak{K}_{2}$, $U f \in \mathbb{R}_{2}(\lambda) \subset \mathfrak{K}_{2}$. If we represent $U$ as a matrix, $U \sim\left(U_{j_{k}}\right)$, where $U_{j k}$ maps $\mathfrak{S}_{k}$ into $\mathfrak{K}_{j}$, then it follows that $U_{12} \equiv 0$ on $\mathfrak{K}_{2}$. Further, $U f=U_{22} f$ for all $f \in \mathfrak{S}_{2}$, so that $U_{22}$ maps $\mathfrak{S}_{2}$ isometrically onto $\mathfrak{R}_{2}(\lambda)$. $U_{22}$ is thus a partial isometry in $\mathfrak{S}_{2}$ with initial set $\mathfrak{S}_{2}$ and final set $\mathfrak{R}_{2}(\lambda)$, while $U_{22}^{*}$ is a partial isometry with initial set $\mathfrak{R}_{2}(\lambda)$ and final set $\mathfrak{K}_{2}$. We have that $E=P\left(\mathfrak{K}_{2}\right)=U_{22}^{*} U_{22}$, while $P\left(\Re_{2}(\lambda)\right)=U_{22} U_{22}^{*}$.

Now let $A$ be any bounded operator in $\mathfrak{S}$ with matrix representation

$$
A \sim\left(\begin{array}{ll}
E & B \\
B^{*} & C
\end{array}\right),
$$

where $E$ is the identity in $\mathfrak{S}_{1}, B$ maps $\mathfrak{S}_{2}$ into $\mathfrak{S}_{1}, C$ maps $\mathfrak{S}_{2}$ into $\mathfrak{S}_{2}$, and $C$ is self-adjoint. Suppose that $A$ commutes with $H$. We shall show that this implies $B \equiv 0$. Then by the proposition of M. A. Naimark [6] mentioned in the introduction, it follows that the spectral function $E_{1}(\lambda)$ defined by $H$ is extremal.

Since $A$ commutes with $H$, it commutes with the Cayley transform $U$ of $H$. This implies that $B U_{21}=U_{12} B^{*}$ and $U_{12}+B U_{22}=$ $U_{11} B+U_{12} C$. Since $U_{12} \equiv 0$, these equations become $B U_{21} \equiv 0$ and $B U_{22}=U_{11} B$. On $\mathfrak{M}_{1}(\bar{\lambda}), \quad U_{21}=V_{21}$ and therefore $B V_{21} \mathfrak{M q}_{1}(\bar{\lambda})=$ $B U_{21} \mathfrak{M R}_{1}(\bar{\lambda})=\{0\} . \quad$ Becaese by Theorem 2, $V_{21} \mathfrak{M M}_{1}(\bar{\lambda})$ is dense in $\mathfrak{M R}_{2}(\lambda)$, $B \mathfrak{M}_{2}(\lambda)=\{0\}$, i.e., $B P\left(\mathfrak{M}_{2}(\lambda)\right)=0$. From the equation $B U_{22}=U_{11} B$ we have that $B P\left(\Omega_{2}(\lambda)\right)=B U_{22} U_{22}^{*}=U_{11} B U_{22}^{*}$. Adding $B P\left(\Omega_{2}(\lambda)\right)=$ $U_{11} B U_{22}^{*}$ with $B P\left(\mathfrak{M R}_{2}(\lambda)\right)=0$, we obtain that $B=U_{11} B U_{22}^{*}$. By iterating this equation we see that $B=U_{11}^{p} B\left(U_{22}^{*}\right)^{p}$ for every positive integer p. Since $\left\|U_{11}\right\| \leqq 1,\|B f\| \leqq\|B\|\left\|\left(U_{22}^{*}\right)^{p} f\right\|$ for each $f \in \mathfrak{S}_{2}$ and each positive integer $p$. 
By Theorem $8, \mathfrak{R}_{2}(\lambda)=\mathfrak{M}^{\prime} \oplus \mathfrak{M}^{\prime \prime}$, where $U_{22}^{*}$ maps $\mathfrak{M}^{\prime \prime}$ isometrically onto $\mathfrak{M}^{\prime \prime}$, and if $f \in \mathfrak{M}_{2}(\lambda) \oplus \mathfrak{M}^{\prime}$, then $\lim _{p \rightarrow \infty}\left\|\left(U_{22}^{*}\right)^{p} f\right\|=0$. But if $U_{22}^{*}$ maps $\mathfrak{M}^{\prime \prime}$ isometrically onto $\mathfrak{M}^{\prime \prime}$, then $U_{22}$ and therefore $U$ maps $\mathfrak{M}^{\prime \prime}$ isometrically onto $\mathfrak{M}^{\prime \prime}$. This means that $U$ and therefore $H$ is reduced by $\mathfrak{M}^{\prime \prime}$, a subspace of $\mathfrak{K}_{2}$. Since $H$ is a minimal self-adjoint extension of $H_{1}, \mathfrak{M}^{\prime \prime}=\{0\}$. Hence, $\mathfrak{L}_{2}=\mathfrak{M}_{2}(\lambda) \oplus \mathfrak{M}^{\prime}$, and thus if $f \in \mathfrak{S}_{2}$, $\lim _{p \rightarrow \infty}\left\|\left(U_{22}^{*}\right)^{p} f\right\|=0$. Since $\|B f\| \leqq\|B\|\left\|\left(U_{22}^{*}\right)^{p} f\right\|$ for each $f \in \mathfrak{K}_{2}$ and for every positive integer $p$, it follows that $B \equiv 0$ on $\mathfrak{K}_{2}$. This completes the proof of Theorem 9 .

Since the operator $H_{2}$ in Theorem 9 is a Hermitian operator with deficiency indices $(0, s)$ or $(s, 0)$, it may seem that we are dealing with a wider class of operators than the maximal symmetric operators. That this is not so is shown by Theorem 10 below.

THEOREM 10. If $H$ is a Hermitian operator with deficiency indices $(0, s)$ or $(s, 0)$, then $H$ is a maximal symmetric operator. If $H$ is a Hermitian operator with deficiency indices $(0,0)$, then $H$ is a self-adjoint operator.

Proof. If $H$ is a Hermitian operator and $\mathfrak{B}=\mathfrak{S} \ominus[\mathfrak{D}(H)]^{c}$, then $\mathfrak{B} \cap \mathfrak{L}(\bar{\lambda})=\{0\} . \quad$ (If $h \in \mathfrak{B} \cap \mathfrak{Q}(\bar{\lambda})$, then $h=(H-\lambda E) g$, Hence, $0=$ $(h, g)=(H g, g)-\lambda(g, g)$. Since $(H g, g)$ is real while $\lambda$ is not, $g=0$. This simple argument is due to M. A. Krasnosel'skii [2, Lemma 2].) If $H$ has deficiency indices $(0, s), \mathfrak{M}(\bar{\lambda})=\{0\}$ so that $\mathfrak{B} \subset \mathfrak{L}(\bar{\lambda})$. Thus, $\mathfrak{B}=\{0\}$ and $H$ is symmetric. Similarly, $H$ is symmetric if its deficiency indices are $(s, 0)$. It follows immediately that if $H$ has deficiency indices $(0,0), H$ is self-adjoint. Theorem 10 is proved.

\section{REFERENCES}

1. N. I. Achieser and I. M. Glasmann, Theorie der linearen operatoren im HilbertRaum, Akademie-Verlag, Berlin, 1954.

2. M. A. Krasnosel'skii, On self-adjoint extensions of Hermitian operators, Ukrainskii Mat. Zhurnal, No. 1 (1949), 21-38.

3. F. J. Murray and J. v. Neumann, On rings of operators, Annals of Math., 37 (1936), 116-229.

4. Bela v. Sz. Nagy, Spektraldarstellung linearer transformationen des Hilbertschen. Raumes, Springer Verlag, Berlin, 1942.

5. M. A. Naimark, Spectral functions of a symmetric operator, Izvest. Akad. Nauk SSSR, Ser. Mat., 4 (1940), 277-318.

6. - Extremal spectral functions of a symmetric operator, Izvest. Akad. Nauk. SSSR, Ser. Mat., 11 (1942), 327-344.

Mathematics Research Center, U.S. ARmy

UNIVERSITY OF WISCONSIN, AND

UNIVERSITY OF CALIFORNIA, RIVERSIDE 


\section{PACIFIC JOURNAL OF MATHEMATICS}

\section{EDITORS}

Robert Osserman

Stanford University

Stanford, California

M. G. Arsove

University of Washington

Seattle 5 , Washington
J. DugundjI

University of Southern Califorma: Los Angeles 7, California

Lowell J. Paige

University of California

Los Angeles 24, California

\section{ASSOCIATE EDITORS}

E. F. BECKENBACH

B. H. NeumanN

F. WOLF

K. YosIDA

\section{SUPPORTING INSTITUTIONS}

UNIVERSITY OF BRITISH COLUMBIA

CALIFORNIA INSTITUTE OF TECHNOLOGY

UNIVERSITY OF CALIFORNIA

MONTANA STATE UNIVERSITY

UNIVERSITY OF NEVADA

NEW MEXICO STATE UNIVERSITY

OREGON STATE UNIVERSITY

UNIVERSITY OF OREGON

OSAKA UNIVERSITY

UNIVERSITY OF SOUTHERN CALIFORNIA
STANFORD UNIVERSITY

UNIVERSITY OF TOKYO

UNIVERSITY OF UTAH

WASHINGTON STATE UNIVERSITY

UNIVERSITY OF WASHINGTON

AMERICAN MATHEMATICAL SOCIETY CALIFORNIA RESEARCH CORPORATION SPACE TECHNOLOGY LABORATORIES NAVAL ORDNANCE TEST STATION 


\section{Pacific Journal of Mathematics}

\section{Vol. 14, No. 1 \\ May, 1964}

Richard Arens, Normal form for a Pfaffian .........................

Charles Vernon Coffman, Non-linear differential equations on cones in Banach

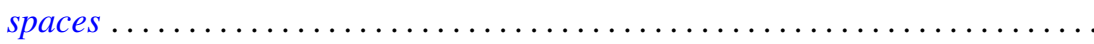

Ralph DeMarr, Order convergence in linear topological spaces ..............

Peter Larkin Duren, On the spectrum of a Toeplitz operator ................

Robert E. Edwards, Endomorphisms of function-spaces which leave stable all

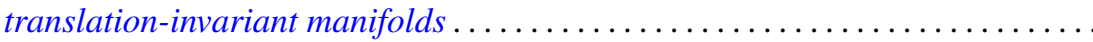

Erik Maurice Ellentuck, Infinite products of isols . . . . . . . . . . . . . . . . 49

William James Firey, Some applications of means of convex bodies . . . . . . . . 53

Haim Gaifman, Concerning measures on Boolean algebras ............. 61

Richard Carl Gilbert, Extremal spectral functions of a symmetric operator. . . . . . 75

Ronald Lewis Graham, On finite sums of reciprocals of distinct nth powers ..... 85

Hwa Suk Hahn, On the relative growth of differences of partition functions ...... 93

Isidore Isaac Hirschman, Jr., Extreme eigen values of Toeplitz forms associated

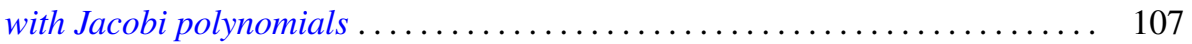

Chen-jung Hsu, Remarks on certain almost product spaces . . . . . . . . . . . 163

George Seth Innis, Jr., Some reproducing kernels for the unit disk . . . . . . . . . 177

Ronald Jacobowitz, Multiplicativity of the local Hilbert symbol . . . . . . . . . . . 187

Paul Joseph Kelly, On some mappings related to graphs ................. 191

William A. Kirk, On curvature of a metric space at a point . . . . . . . . . . . . 195

G. J. Kurowski, On the convergence of semi-discrete analytic functions . . . . . . . 199

Richard George Laatsch, Extensions of subadditive functions . . . . . . . . . . . 209

V. Marić, On some properties of solutions of $\Delta \psi+A\left(r^{2}\right) X \nabla \psi+C\left(r^{2}\right) \psi=0 \ldots 217$

William H. Mills, Polynomials with minimal value sets . . . . . . . . . . . 225

George James Minty, Jr., On the monotonicity of the gradient of a convex

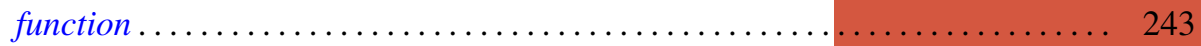

George James Minty, Jr., On the solvability of nonlinear functional equations of 'monotonic' type ................................... 249

J. B. Muskat, On the solvability of $x^{e} \equiv e(\bmod p) \ldots \ldots \ldots \ldots \ldots \ldots \ldots \ldots . \ldots \ldots$

Zeev Nehari, On an inequality of $P . R$. Bessack ................... 261

Raymond Moos Redheffer and Ernst Gabor Straus, Degenerate elliptic

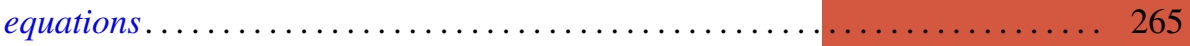

Abraham Robinson, On generalized limits and linear functionals . . . . . . . . . 269

Bernard W. Roos, On a class of singular second order differential equations with a

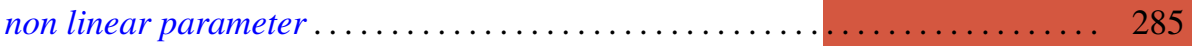

Tôru Saitô, Ordered completely regular semigroups . . . . . . . . . . . . . . . . 295

Edward Silverman, A problem of least area ....................... 309

Robert C. Sine, Spectral decomposition of a class of operators . . . . . . . . . 333

Jonathan Dean Swift, Chains and graphs of Ostrom planes . . . . . . . . . . . 353

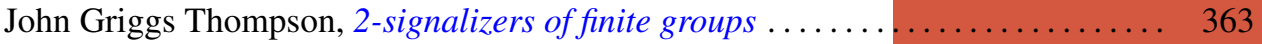

Harold Widom, On the spectrum of a Toeplitz operator . . . . . . . . . . . . . 365 